\title{
Physiologic Brain Dysmaturity in Late Preterm Infants
}

\author{
MARK S. SCHER, MARK W. JOHNSON, SUSAN M. LUDINGTON, AND KENNETH LOPARO \\ Departments of Pediatric Neurology [M.S.S.], Pediatric Medicine [M.W.J.], and Electrical Engineering and Computer Science [K.L.], \\ Case Western Reserve University School of Medicine, Cleveland, Ohio 44106
}

\begin{abstract}
Neonatal EEG sleep was used to determine whether differences are expressed between healthy late preterm and full-term (FT) groups. Twenty-seven 24-channel multihour studies were recorded at similar postmenstrual ages (PMA) and analyzed for eight asymptomatic late preterm infants (LPT) compared with 19 healthy FT infants as a preliminary analysis, followed by a comparison of a subset of eight FT infants, matched for gender, race, and PMA. $Z$ scores were performed on data sets from each group pair comparing each of seven EEG/Sleep measures for entire recordings, active sleep (AS) and quiet sleep (QS) segments and artifact-free intervals. Six of seven measures showed differences between the eight LPT and eight matched FT cohort pair comparisons of $>0.3$; rapid eye movements, arousals during QS, spectral correlations between homologous centrotemporal regions during QS, spectral beta/alpha power ratios during AS and QS, a spectral measure of respiratory regularity during QS, and sleep cycle length. Quantitative neurophysiologic analyses define differences in brain maturation between LPT and FT infants at similar PMA. Altered EEG/Sleep behaviors in the LPT are biomarkers of developmental neuroplasticity involving interconnected neuronal networks adapting to conditions of prematurity for this largest segment of the preterm neonatal population. (Pediatr Res 70: 524-528, 2011)
\end{abstract}

$\mathrm{P}$ reterm infants comprise $12.8 \%$ of all live births with the late preterm neonate (LPT) making up $72 \%$ of the overall preterm population (1). The birth rate for LPT (i.e. 34 0/7 to 36 6/7 wk) has been steadily increasing over the last quarter century. Although the number of neonates $<34$ wk has increased by approximately $10 \%$ since 1990, LPT has increased by nearly $25 \%$. Although more mature preterm infants have historically been considered healthy with comparatively low risk, there is now growing evidence that this population is not as healthy as previously considered. Compared with full-term (FT) infants, there is an increased mortality as well as higher risks for complications such as transient tachypnea of the newborn (TTN), RDS, persistent pulmonary hypertension (PPHN), respiratory failure, temperature instability, jaundice, sepsis, feeding difficulties, and prolonged NICU stays. Given their greater numbers compared with all preterm infants, LPT consume a significant amount of health care resources. In addition to increased mortality, LPT have an increased likelihood of neurological morbidities leading to adverse long-

Received January 6, 2011; accepted June 3, 2011

Correspondence: Mark S. Scher, MD, Pediatric Neurology, Rainbow Babies and Children's Hospital, University Hospitals of Cleveland, Case Western Reserve University School of Medicine, 11100 Euclid Avenue, MS6090, Cleveland, OH 44106; e-mail: mark.scher@uhhospitals.org

Supported, in part, by National Institutes of Health grants, NS26793, RR 00084, NR01894, NS34508 [to M.S.S.], and NR 04926 [to S.M.L.].

The authors report no conflicts of interest. term neurodevelopmental consequences. Reliable neonatal biomarkers are needed that can more specifically define neurological status for LPT as a function of postnatal brain organization and maturation to help more accurately track neurodevelopmental outcome after the institution of neuroprotective interventions.

Functional brain organization and maturation of newborns have been assessed by visual and digital analyses of EEG/Sleep differences for over the past 50 years (2). This bedside neurophysiological test remains a reliable and comparatively low-cost biomarker of brain function for different neonatal populations. Differences in EEG/Sleep organization and maturation have been described between more immature preterm $(<32 \mathrm{wk} \mathrm{GA})$ and FT cohorts at matched postmenstrual ages (PMA) (3-9). Specific EEG/Sleep behaviors suggest either an acceleration of brain maturation as expected for an older infant when compared with the FT neonate. Other measures suggest a delay in brain maturation expected for a preterm neonate $(10,11)$. To reconcile physiologic precocity or immaturity for any particular EEG/Sleep measure, an analytic approach comparing Mahalonobis distances were performed between preterm and FT cohorts across different physiologic groupings of multiple EEG/Sleep behaviors. A physiologic dysmaturity index (DI) was then defined based on seven measures of neonatal EEG/Sleep that best differentiated these neonatal cohorts in terms of state-specific neurophysiologic behaviors. DI was initially described for a healthy preterm group born less than 32-wk GA for a comparison to EEG/Sleep behaviors of term infants at matched postmenstrual term ages. This study compares the DI of a LPT cohort to a FT group.

\section{METHODS}

Patient selection. The Institutional Review Boards (IRB) at Rainbow Babies and Children's Hospital, University Hospitals of Cleveland, Case Western Reserve University (Cleveland, OH), and Magee-Women's Hospital, University of Pittsburgh (Pittsburgh, PA) approved consent forms used to recruit subjects for this study. These 27 neonates were part of a larger database of 461 subjects and 1116 multihour recordings. Clinical, demographic, and neurophysiologic information for this specific cohort was extracted from this larger database. Recruitment over a 20-year period of all subjects in the database were part of five National Institutes of Healthsupported research grants, with IRB-approved consent forms, identified from neonatal populations admitted to the two NICUs of these two hospitals. All EEG/Sleep studies were individually analyzed by visual and digital analysis methodologies.

\footnotetext{
Abbreviations: AS, active sleep; DI, dysmaturity index; FT, full-term; LPT, late preterm infants; NBRS, Nursery Neurobiologic Risk Score; PMA, postmenstrual age; PT, preterm; QS, quiet sleep; REM, rapid eye movement
} 
From the total research group, eight LPT born between 34 0/7 and 36 6/7 wk GA were identified. Selection was based on review of maternal, fetal, and neonatal medical records, and recruitment occurred after consultations with the attending neonatologist. Infants chosen for this group were clinically asymptomatic throughout the study period. None were treated for severe, sepsis, encephalopathy, or seizures. Brazy Neurobiologic Risk Scores (NBRS) had been historically used for the entire preterm cohort in our database as this scoring system is designed for LBW infants to predict neurodevelopmental outcome. For the present study, as our study group was LPT, we only adopted the severity of illness score at FT ages to confirm healthy medical status for the LPT group to then compare with a healthy FT group without the intention of comparing later outcome. NBRS consists of a score of 1 to 4 for each of seven clinical items: blood $\mathrm{pH}$, hypoglycemia, intraventricular hemorrhage, periventricular leukomalacia, seizures, infection, and the need for mechanical ventilation. Normal cranial ultrasounds were therefore described for all preterm neonates. Scores can be classified as low risk $(\leq 4)$, moderate risk $(5-7)$, and high risk $(\geq 8)(12)$ and were all low risk for the LPT group. The PMA listed for each subject was the corrected age at term when the EEG/Sleep study was performed.

Nineteen appropriate for GA FT infants were identified from the database. Reviews of maternal and neonatal records as well as physical examinations were performed to verify the healthy status of this group. Nineteen neonates were initially identified whose EEG/Sleep measures were compared with the study group, matched over a wider GA range of 38 to $42 \mathrm{wk}$ (mean group difference of $2 \mathrm{wk}$ ), irrespective of gender or race. As with the LPT group, the PMA for each FT subject was the age when the EEG/Sleep study was performed. A group of eight FT newborns were then selected who were matched for gender, race, and a narrower PMA range (mean difference of $6 \mathrm{~d}$ ). For the eight LPT and eight matched comparisons, all subjects were Caucasian.

EEG sleep recordings. Electroencephalographic/polysomnographic studies (EEG/Sleep) were recorded on multimodality 24-channel recording devices (Nihon-Kohden America, Inc., Foothill Ranch, CA; model 4221) and carried out in an environmentally controlled setting in which sound, light, humidity, and tactile stimulation were monitored, as discussed in previously publications (4). All infants were studied while sleeping prone or on their sides in an open bed which was their usual sleeping position in the nursery. Continuous recordings for $3 \mathrm{~h}$ began after a diaper change and feeding at 0900 to $1000 \mathrm{~h}$ and ended between 1200 and $1300 \mathrm{~h}$ on the same day.

Off-line visual analyses of the all digitized neurophysiologic data were performed. Typed comments and start/end cursors were electronically recorded for each multihour recording. All notations were then tabulated to derive numbers and durations for specific EEG/Sleep measures; sleep state durations, arousals, and rapid eye movements (REMs). One of six neonatal sleep state segments was assigned by a single neurophysiologist (M.S.S.) according to conventional neonatal EEG sleep criteria $(2,13)$. Segments included two active sleep (AS) and two quiet sleep (QS) segments as well as indeterminate sleep and waking intervals. For the purposes of this publication, each of the two AS and QS segments were combined into one AS and QS length in minutes. All minutes of all EEG/Sleep studies were scored by M.S.S., including start and end times of physiologic arousals during all consecutive minutes of the recording. Sleep state segments were digitally annotated on a display of the EEG recording with a start and end time. Artifact segments primarily containing excessive movement-induced artifact during which a sleep state could not be determined were identified. These epochs were later electronically removed to obtain artifact-free segments of all EEG/Sleep segments for each study while preserving consecutive minutes of sleep that included transient arousal periods. Artifact-free segments of AS and QS permitted more accurate comparisons between groups for spectral power, correlations, and respiratory regularity as well as comparisons of arousal indices and REM, as excessive periods of muscle activity and movements would alter these spectral measures. REMs were visually identified on the EOG channels, and electronic cursors were placed on the record to obtain totals per unit time.

A neonatal research nurse provided clinical care for each infant during the recording session. Sleep, feeding, behavior, diaper changes, medication administration, and technical comments (i.e. equipment malfunctions and environmental measures) were documented in the computer database provided for these studies. Light and sound levels were continuously recorded and entered into the data files. No infants were given medications during the studies. No male children were circumcised before the study.

EEG sleep measures. A previously described DI consisting of seven EEG/Sleep measures has been historically used to quantify physiologic differences in brain organization and maturation between healthy preterm infants $<32$ wk gestation and a FT group at matched PMA $(5-8,10,11)$. The seven EEG sleep measures were arousal numbers, REM numbers, percentage of QS, sleep cycle length, spectral beta/alpha EEG energy ratios, spectral EEG correlations between left and right hemispheric centrotemporal regions (i.e.
$\mathrm{T} 3 \mathrm{C} 3 / \mathrm{C} 4 \mathrm{~T} 4$ ), and a spectral measure of respiratory regularity. These measures were statistically selected from initial physiologic groupings of 34 EEG/Sleep measures. Each of the seven measures best represent the interconnected neuronal circuitries expressed as precocious or immature neurophysiologic behaviors of a preterm cohort when compared with FT infants $(4,10,11,14)$ at similar PMA.

Analytic methods. The ultimate goal for this study was to compare an effect size by Z-score comparisons between LPT and FT cohorts. Exploratory graphical methods demonstrated that distributions of measures for both cohorts were well represented by normal Gaussian distributions.

Standard Z-scores were calculated as follows: differences between the means of the cohorts over the pooled SDs. Subanalyses were included to help verify true differences between cohorts rather than by chance. Effect sizes were also assessed for both total and artifact-free minutes during active and QS segments, identified by visual analyses. Z-score values of 0.3 or greater for the eight LPT and eight matched FT comparisons were considered supportive of our hypothesis for this publication that physiologic dysmaturity exists in healthy LPT in comparison to FT infants. This threshold for the $Z$ score was chosen because a significant $\mathrm{p}$-value of at least 0.05 could be predicted if greater numbers of subjects were recruited.

\section{RESULTS}

Demographic features. Table 1 presents the eight subjects in the LPT group compared with 19 and eight matched FT comparison groups with respect to similar PMA at the time of the study and low risk NBRS of $<4$. Birth weight, height, and head circumferences at birth and at the time of the study at term age are listed, including percentiles for these measurements. Along with the 19 FT cohort, eight neonates were also included in Table 1, matched for gender, race, and a more similar PMA (i.e. mean $6 \mathrm{~d}$ ).

The LPT cohort had smaller measurements for head circumference, height, and weight (means, SDs, and percentiles

Table 1. Subject demographics and measurements

\begin{tabular}{lccc}
\hline \multicolumn{1}{c}{ Cohort } & Late preterm & Full term* & Full term \\
\hline $\begin{array}{l}\text { Subjects }(N) \\
\text { Sex }\end{array}$ & 8 & 8 & 19 \\
$\quad$ Female & 4 & 4 & 9 \\
$\quad$ Male & 4 & 4 & 10 \\
Race & & & \\
$\quad$ African American & 0 & 0 & 4 \\
$\quad$ Caucasian & 8 & 8 & 15 \\
Birth statistics & & & \\
$\quad$ GA (wk) & & & \\
$\quad$ Length $(\mathrm{cm})$ & $35.5 \pm 0.6$ & $38.3 \pm 1.1$ & $38.7 \pm 1.2$ \\
$\quad$ Weight $(\mathrm{kg})$ & $43.4 \pm 4.0$ & $49.4 \pm 2.9$ & $49.9 \pm 3.9$ \\
$\quad$ Head circumference $(\mathrm{cm})$ & $30.9 \pm 2.1$ & $34.6 \pm 2.1$ & $34.3 \pm 2.4$ \\
\end{tabular}

Average birth percentiles for GA

Length (\%)

Weight $(\%)$

Head circumference $(\%)$

$21.1 \pm 34.4$

$16.2 \pm 23.3$

$42.5+35.1$

$49.2 \pm 36.6$

EEG study age statistics

PMA (wk)

$28.2 \pm 33.2$

$52.1+32$.

$58.2 \pm 34.2$

Length $(\mathrm{cm})$

Weight $(\mathrm{kg})$

$38.7 \pm 0.7$

$51.3 \pm 32.0$

$46.3 \pm 33.5$

Head circumference $(\mathrm{cm})$

Average study percentiles

Length (\%)

$45.4 \pm 4.1$

$39.5 \pm 1.1$

$40.7 \pm 1.6$

$2.32 \pm 0.77$

$49.6 \pm 3.4$

$51.1 \pm 4.2$

$32.7 \pm 2.6$

$3.48 \pm 0.71$

$3.65 \pm 0.81$

Weight (\%)

$13.4 \pm 21.5$

$35.7 \pm 1.5$

$35.9 \pm 2.0$

Head circumference $(\%)$

$11.6 \pm 20.0$

$35.2 \pm 34.2 \quad 46.7 \pm 37.3$

Values are count or mean $\pm \mathrm{SD}$

* Full-term subset matched to late-preterm group by gender, race, and PMA at EEG study.

Brazy Neurobiologic Risk Score (NBRS) was less than 4 for all subjects in all groups. 
Table 2. Summary of late preterm vs full-term effect sizes [Z-scores] for dysmaturity index parameters

\begin{tabular}{|c|c|c|c|}
\hline Cohort & Late preterm & Full term & Full term* \\
\hline GA range (wk) & 34 to $366 / 7$ & $\geq 37$ & $\geq 37$ \\
\hline Subject count & 8 & 19 [8 vs 19] & $8[8$ vs 8$]$ \\
\hline Active sleep REM index & $3.9 \pm 2.3$ & $5.4 \pm 2.5[-0.59 \dagger]$ & $5.4 \pm 2.7[-0.57 \dagger]$ \\
\hline Quiet sleep arousals index & $4.1 \pm 2.2$ & $4.1 \pm 1.9[0.02]$ & $4.8 \pm 2.2[-0.30 \dagger]$ \\
\hline Percentage quiet sleep & $34.4 \pm 8.5$ & $36.9 \pm 9.2[-0.28 \dagger]$ & $35.5 \pm 11.7[-0.10]$ \\
\hline Sleep cycle length & $62.0 \pm 19.8$ & $65.7 \pm 22.0[-0.17]$ & $70.0 \pm 28.7[-0.32 \dagger]$ \\
\hline \multicolumn{4}{|l|}{ Full study (all epochs) } \\
\hline Left/right pair correlation & $0.12 \pm 0.04$ & $0.17 \pm 0.09[-0.67 \dagger]$ & $0.16 \pm 0.07[-0.80 \dagger]$ \\
\hline Beta/alpha ratio index & $-0.12 \pm 0.04$ & $-0.15 \pm 0.07[0.36 \dagger]$ & $-0.11 \pm 0.07[-0.25]$ \\
\hline Respiratory regularity index & $-0.93 \pm 0.09$ & $-1.00 \pm 0.13[0.57 \dagger]$ & $-0.96 \pm 0.13[0.30 \dagger]$ \\
\hline \multicolumn{4}{|l|}{ Arousal-free quiet sleep epochs } \\
\hline Left/right pair correlation & $0.15 \pm 0.07$ & $0.21 \pm 0.13[-0.49 \dagger]$ & $0.18 \pm 0.09[-0.32 \dagger]$ \\
\hline Beta/alpha ratio index & $-0.21 \pm 0.07$ & $-0.20 \pm 0.08[-0.20]$ & $-0.15 \pm 0.06[-0.97 \dagger]$ \\
\hline Respiratory regularity index & $-1.20 \pm 0.11$ & $-1.21 \pm 0.14[0.10]$ & $-1.17 \pm 0.14[-0.28 \dagger]$ \\
\hline \multicolumn{4}{|l|}{ Arousal-free active sleep epochs } \\
\hline Left/right pair correlation & $0.18 \pm 0.07$ & $0.19 \pm 0.12[-0.12]$ & $0.18 \pm 0.06[-0.01]$ \\
\hline Beta/alpha ratio index & $-0.05 \pm 0.05$ & $-0.12 \pm 0.08[0.89 \dagger]$ & $-0.09 \pm 0.07[0.56 \dagger]$ \\
\hline Respiratory regularity index & $-0.84 \pm 0.10$ & $-0.92 \pm 0.14[0.65 \dagger]$ & $-0.90 \pm 0.12[0.58 \dagger]$ \\
\hline
\end{tabular}

Values are mean \pm SD and [Z-score]. Brazy Neurobiologic Risk Score (NBRS) was $<4$ for all subjects in all groups. Effect sizes [Z-scores] are the (difference between the means)/(pooled SD).

* Full-term subset matched to late-preterm group by gender, race, and PMA at EEG study.

$\dagger$ Magnitude of Z-scores $>0.25$.

listed in Table 1), both at birth and when compared with FT cohorts.

EEG/Sleep measures. Table 2 lists the effect sizes or $Z$-scores between cohorts for the seven DI measures. Z-score comparisons are listed for the eight LPT versus 19 FT neonates as well as for the eight LPT versus eight matched FT. Five of the seven DI measures showed effect size differences of 0.25 or greater between the eight and 19 groups, including REM, \% QS, spectral measures of correlation, beta/alpha ratios, and respiratory regularity. Six of seven of the DI measures showed effect sizes of 0.30 or greater for the eight and eight cohort comparisons, including REM, QS arousals, sleep cycle length, spectral measures of correlation, beta/alpha ratio, and respiratory regularity. When artifact-free epochs were compared, both the eight and 19 and eight and eight comparisons had significant differences for spectral correlation, AS spectral beta/alpha ratios, and AS respiratory regularity. However, only the eight LPT and eight matched FT group comparisons resulted in significant differences during QS for spectral beta/alpha ratios and respiratory regularity.

\section{DISCUSSION}

The brain volume of a late preterm infant (LPT) at $34 \mathrm{wk}$ gestation is approximately $65 \%$ of the term brain (15). There will be a 5 -fold increase in white matter volume between 35 and 41 wk gestation. Important maturational changes occur within the brain during late preterm gestation that include increasing neuronal connectivity, dendritic arborization, and synaptic junctions, as well as maturation of neurochemical and enzymatic processes that take part in the regulation of brain growth and maturation. Although less common than younger preterm infants, LPT are more likely to develop periventricular leukomalacia than FT infants because of white matter vulnerability under adverse conditions. LPT are consequentially more vulnerable to more remote injuries in cortical and subcortical gray matter in regions where damaged white matter tracts innervate. Combined white and gray matter injuries lead to neurocognitive and behavioral deficits as the brain matures throughout childhood, as reported for LPT. Neonatal biomarkers are needed that can predict these deficits and quantify the effects of neuroprotective interventions.

Quantitative EEG/Sleep studies in LPT have not been specifically reported, although LPT is historically referred to as a cohort which displays EEG/Sleep activities that may resemble either preterm or FT patterns (2). This cohort is generally assumed to express specific physiologic behaviors that are intermediate between infants born before $34 \mathrm{wk}$ and those that are near or at term. This has been pointed out to be generally true for the control of breathing, heart rate, and sleep state maturation $(16,17)$. There is a delay in maturation of integrated autonomic brainstem function throughout the neuroaxis which contributes to an increased risk for acute lifethreatening events including sudden infant death syndrome compared with term infants.

To our knowledge, our study is the first to report altered quantitative EEG/Sleep in LPT using visually scored and spectrally calculated neurophysiologic measures. Although there have been maturational studies demonstrating differences in EEG/Sleep in preterm infants $<32$ wk gestation $(2,9,18)$, we now suggest that LPT uniquely express altered functional neuronal networks that differ from FT infants. Our study suggests that the DI derived from an EEG/Sleep study can be a meaningful physiologic biomarker of functional brain organization and maturation applicable to a LPT population. These physiologic differences may influence how the LPT will react to prenatal/postnatal illnesses, environmental stresses, and neuroprotective interventions with long-term neurodevelopmental consequences.

EEG/Sleep behaviors are physiologic surrogates of multiple interconnected neuronal pathways that course throughout the 
neuroaxis within brainstem, diencephalic, and cortical structures. Connectivities among specific pathways subserve state regulation (19-21). The pontomedullary to basal-frontal pathways subserve respiratory activity. The penduculopontine geniculocalacarine pathways are identified with REM behavior. The ascending reticular activating pathway subserves arousals. The corticothalamic pathways subserve QS (nonREM) expression and the corticocortical pathways are expressed as spectral beta/alpha energy ratios and electrode-pair correlations. Our study suggests that for LPT, these neuronal networks mature differently compared with a FT infant and express unique state-specific EEG/Sleep behaviors from its FT counterpart.

We noted different results in EEG/Sleep expression between LPT and FT groups when comparing the two groups pairs, suggesting that a greater range of PMA (mean of $2 \mathrm{wk}$ ) as well as differences in race and gender for the eight and 19 cohort comparisons influence specific neurophysiologic behaviors. Spectral correlation and beta/alpha ratios may be more robust measures reflecting less developed brain organization and maturation in LPT compared with FT for both group pair comparisons despite greater variations in PMA, gender, and race for the eight and 19 cohort pair. However, greater variations in PMA, gender, and race may better explain differences in \% QS and respiratory regularity (the latter calculated over the full study) rather than denoting specific differences between LPT and FT EEG/Sleep, as these findings are no longer significant in the eight LPT and eight matched FT comparison. When there is a closer match in PMA (mean of less than $6 \mathrm{~d}$ ), gender, and race with the eight and eight cohort comparison, other significant EEG/Sleep differences emerge. More mature (i.e. fewer) arousals but more immature (i.e. shorter) sleep cycle lengths are noted in the LPT group. QS-specific differences between LPT and FT are also observed. This tendency to observe differences for specific EEG/ Sleep measures between LPT and FT neonatal groups during QS has been previously observed in a cohort of healthy neonates over successive days of life after neonatal depression from a difficult parturition and delivery (22). In general, environmental stress alters sleep architecture, with greater percentages of QS. As a result of the greater stress of longer extrauterine life, the brain of the LPT adapts by expressing more discernible physiologic differences during QS, which is the segment of sleep which may be more responsive to stress factors. What is more, these differences were more easily demonstrated when muscle activity and body movements were removed during artifact-free sleep segments because artifact alters the brain generated spectral profiles expressed as power, correlation, and respiratory regularity.

Our previously reported index of physiologic brain dysmaturity (DI) for a healthy preterm cohort (i.e. $<32 \mathrm{wk}$ GA) at term compared with a FT cohort can be applied as a quantitative metric to represent the interconnected pathways expressed during sleep in LPT. This DI was statistically derived from winnowing down from 34 to seven physiologic measures of EEG/Sleep that best differentiate brain organization and maturation between cohorts $(4,5,11)$. The present study demonstrates that six of these seven measures also differentiate differences in these neuronal networks for LPT compared with FT cohorts, when controlling for PMA, race, and gender for the eight and eight cohort pair comparison. We plan additional studies of LPT to further investigate time-dependent nonlinear and neuronal connectivity relationships, as we previously reported in younger preterm cohorts, to study brain maturation using specific algorithms that quantify neuronal network complexity (23-25).

Previously described as ontogenetic adaptation (26), this biological process of developmental neuroplasticity has been redefined in terms of genetic and epigenetic variability at molecular, cellular, and neuronal network levels of biological function (23). Quantitative neurophysiologic measures such as defined by the physiologic brain DI represent functional neural network biomarkers of plasticity in developing brain circuitries. EEG/Sleep behaviors in an LPT cohort reflect this adaptive process of neuroplasticity unique to conditions of prematurity for this GA range cohort. Such a metric when combined with genotypic/proteomic biomarkers can help define a functional endophenotype, with greater sensitivity to predict risk for different neurodevelopmental disorders as well as quantify the effects of neuroprotective interventions $(27,28)$. Furthermore, a geneticneurophysiologic endophenotype when combined with neuroimaging biomarkers of the preterm brain (29) will further expand the clinician's structural-functional perspective of the positive and negative consequences of developmental neuroplasticity in preterm populations.

We recognize that the interpretations of our study results are limited by the small sample size and selection strategy of subjects. Repeated assessments with larger sample sizes, matching for multiple demographic and clinical covariates, are needed to validate how universally these biomarkers can be applied to all LPT. For example, we matched for race in the eight LPT and eight matched FT comparison, but all subjects were Caucasian. In addition, although the PMA differences were smaller between the eight LPT and eight FT groups, the LPT group remained approximating $1 \mathrm{wk}$ less mature than the FT group. We also acknowledge that the LPT were smaller in weight, height, and head circumference, reflecting both prenatal and postnatal influences on growth, suggesting that maternal, placental, fetal, and neonatal factors potentially influence brain development through growth mechanisms during prenatal and postnatal periods. Finally, although we used the severity of illness scale, NBRS, as simply an inventory of postnatal diseases, this would be an score not suitable for prediction of neurodevelopmental outcome for LPT. Our results are nonetheless provocative, suggesting that altered neurophysiological maturation exists in LPT, expressed as quantitative measures of EEG/Sleep behaviors. These differences may help predict differences in outcome as well as responses to neuroprotective interventions in future studies.

\section{REFERENCES}

1. Ramachandrappa A, Jain L 2009 Health issues of the late preterm infant. Pediatr Clin North Am 56:565-577

2. Scher MS 2008 Ontogeny of EEG-sleep from neonatal through infancy periods. Sleep Med 9:615-636

3. Scher MS, Dokianakis SG, Sun M, Steppe DA, Guthrie RD, Sclabassi RJ 1996 Computer classification of sleep in preterm and full-term neonates at similar postconceptional term ages. Sleep 19:18-25 
4. Scher MS, Jones BL, Steppe DA, Cork DL, Seltman HJ, Banks DL 2003 Functional brain maturation in neonates as measured by EEG-sleep analyses. Clin Neurophysiol 114:875-882

5. Scher MS, Steppe DA, Dahl RE, Asthana S, Guthrie RD 1992 Comparison of EEG sleep measures in healthy full-term and preterm infants at matched conceptional ages. Sleep 15:442-448

6. Scher MS, Steppe DA, Dokianakis SG, Guthrie RD 1994 Maturation of phasic and continuity measures during sleep in preterm neonates. Pediatr Res 36:732-737

7. Scher MS, Steppe DA, Dokianakis SG, Sun M, Guthrie RD, Sclabassi RJ 1994 Cardiorespiratory behavior during sleep in full-term and preterm neonates at comparable postconceptional term ages. Pediatr Res 36:738-744

8. Scher MS, Sun M, Steppe DA, Banks DL, Guthrie RD, Sclabassi RJ 1994 Comparisons of EEG sleep state-specific spectral values between healthy full-term and preterm infants at comparable postconceptional ages. Sleep 17:47-51

9. Scher MS, Sun M, Steppe DA, Guthrie RD, Sclabassi RJ 1994 Comparisons of EEG spectral and correlation measures between healthy term and preterm infants. Pediatr Neurol 10:104-108

10. Scher MS 1997 Neurophysiological assessment of brain function and maturation. I. A measure of brain adaptation in high risk infants. Pediatr Neurol 16:191-198

11. Scher MS 1997 Neurophysiological assessment of brain function and maturation. II. A measure of brain dysmaturity in healthy preterm neonates. Pediatr Neurol 16:287295

12. Brazy JE, Eckerman CO, Oehler JM, Goldstein RF, O'Rand AM 1991 Nursery Neurobiologic Risk Score: important factor in predicting outcome in very low birth weight infants. J Pediatr 118:783-792

13. Scher MS 2005 Electroencephalography of the newborn: normal and abnormal features. In: Niedermeyer E, Lopes da Silva F (eds) Electroencephalography: Basic Principles, Clinical Applications, and Related Fields. Lippincott Williams \& Wilkins, Philadelphia, PA, pp 937-989

14. Scher MS, Dokianakis SG, Steppe DA, Banks DL, Sclabassi RJ 1997 Computer classification of state in healthy preterm neonates. Sleep 20:132-141

15. Kinney HC 2006 The near-term (late preterm) human brain and risk for periventricular leukomalacia: a review. Semin Perinatol 30:81-88

16. Billiards SS, Pierson CR, Haynes RL, Folkerth RD, Kinney HC 2006 Is the late preterm infant more vulnerable to gray matter injury than the term infant? Clin Perinatol 33:915-933 abstract $\mathrm{x}-\mathrm{x}$
17. Hunt CE 2006 Ontogeny of autonomic regulation in late preterm infants born at 34-37 weeks postmenstrual age. Semin Perinatol 30:73-76

18. Scher MS, Steppe DA, Banks DL, Guthrie RD, Sclabassi RJ 1995 Maturationa trends of EEG-sleep measures in the healthy preterm neonate. Pediatr Neurol $12: 314-322$

19. Datta S, Maclean RR 2007 Neurobiological mechanisms for the regulation of mammalian sleep-wake behavior: reinterpretation of historical evidence and inclusion of contemporary cellular and molecular evidence. Neurosci Biobehav Rev 31:775-824

20. McCarley RW 2007 Neurobiology of REM and NREM sleep. Sleep Med 8:302-330

21. Steriade M 2006 Grouping of brain rhythms in corticothalamic systems. Neuroscience 137:1087-1106

22. Scher MS, Steppe DA, Beggarly ME, Salerno DG, Banks DL 2002 Neonatal EEG-sleep disruption mimicking hypoxic-ischemic encephalopathy after intrapartum asphyxia. Sleep Med 3:411-415

23. Cicchetti D, Blender JA 2006 A multiple-levels-of-analysis perspective on resilience: implications for the developing brain, neural plasticity, and preventive interventions. Ann NY Acad Sci 1094:248-258

24. Janjarasjitt S, Scher MS, Loparo KA 2008 Nonlinear dynamical analysis of the neonatal EEG time series: the relationship between sleep state and complexity. Clin Neurophysiol 119:1812-1823

25. Scher MS, Waisanen H, Loparo K, Johnson MW 2005 Prediction of neonatal state and maturational change using dimensional analysis. J Clin Neurophysiol 22:159165

26. Oppenheim RW 1981 Ontogenetic adaptation and retrogressive processes in the development of the nervous system and behavior: a neuroembryological perspective. In: Connolly KJ, Prechtl HF (eds) Maturation and Development: Biological and Psychological Perspectives. Lippincott Williams \& Wilkins, Philadelphia, PA, pp 73-109

27. Beauchaine TP, Neuhaus E, Brenner SL, Gatzke-Kopp L 2008 Ten good reasons to consider biological processes in prevention and intervention research. Dev Psychopathol 20:745-774

28. Smit DJ, Boersma M, van Beijsterveldt CE, Posthuma D, Boomsma DI, Stam CJ, de Geus EJ 2010 Endophenotypes in a dynamically connected brain. Behav Genet 40:167-177

29. Ment LR, Hirtz D, Huppi PS 2009 Imaging biomarkers of outcome in the developing preterm brain. Lancet Neurol 8:1042-1055 